Research Paper

\title{
Macroscopic types of intrahepatic cholangiocarcinoma and the eighth edition of AJCC/UICC TNM staging system
}

\author{
Ze-Wu Meng ${ }^{1,2, *}$, Wei Pan ${ }^{1,2}$, Hai-Jie Hong ${ }^{1,2}$, Jiang-Zhi Chen ${ }^{1,2}$ and Yan-Ling Chen ${ }^{1,2}$ \\ ${ }^{1}$ Department of Hepatobiliary Surgery, Fujian Medical University Union Hospital, Fuzhou, Fujian 350001, People's Republic \\ of China \\ ${ }^{2}$ Key Laboratory of Ministry of Education for Gastrointestinal Cancer, Fujian Medical University, Fuzhou, Fujian 350001, \\ People's Republic of China \\ *First author
}

Correspondence to: Yan-Ling Chen, email: drchenyl@126.com

Keywords: intrahepatic cholangiocarcinoma, macroscopic type, TNM classification, clinicopathological characteristics, prognosis Received: June 08, $2017 \quad$ Accepted: August 26, $2017 \quad$ Published: September 15, 2017

Copyright: Meng et al. This is an open-access article distributed under the terms of the Creative Commons Attribution License 3.0 (CC BY 3.0), which permits unrestricted use, distribution, and reproduction in any medium, provided the original author and source are credited.

\section{ABSTRACT}

The prognosis of patients with intrahepatic cholangiocarcinoma (ICC) is undefined among the different macroscopic types. This study evaluated the viability of the American Joint Committee on Cancer (AJCC) 8th edition staging classification for different macroscopic types. Utilizing the Surveillance, Epidemiology, and End Results (SEER) database, we enrolled a total of 2,679 eligible patients with an estimated 199 periductal infiltrating type of ICC (ICC-PI) patients and 2,480 mass-forming type of ICC (ICC-MF) patients. After conducting a multivariate Cox analysis, we found that the AJCC 8th edition staging system was suitable for ICC-MF patients but not for ICCPI patients according to cancer-specific survival (CSS) and overall survival (OS). The main reason was the similar hazard ratio (HR) between the ICC-PI patients with stage $I$ and stage II disease according to CSS (HR:0.969, $P=0.949)$ and OS (HR:0.832, $P=0.703$ ). Moreover, we found that ICC-PI patients in AJCC stage I had a similar HR as ICC-MF patients in AJCC stage II according to CSS (HR: 1.208, $P=0.475$ ) and OS (HR:1.206, $P=0.456$ ). Therefore, we suggested that ICC-PI patients may be defined as T2, which is classified as stage II disease. This suggestion for the AJCC 8th edition staging system would be more suitable for different macroscopic types of ICC but requires further verification in prospective clinical trials.

\section{INTRODUCTION}

The incidence and mortality of intrahepatic cholangiocarcinoma (ICC) has prominently increased over the past several decades both in the USA and worldwide [1]. As the second most common liver cancer, ICC is highly malignant and has an extremely poor prognosis [2-5]. The typical mass-forming (MF) type of ICC is a radial growth pattern that invades into the adjacent liver parenchyma, whereas the periductal infiltrating (PI) type of ICC demonstrates a diffuse and often ill-defined longitudinal growth pattern along the bile duct-an obvious clinicopathological difference with MF-type. The prognostic value of the growth pattern remains controversial, and the significance of this variable has not been compared with that of other prognostic factors $[6,7]$.

In the 6th edition staging system for hepatic malignancies established by the American Joint Committee on Cancer (AJCC)/Union for International Cancer Control (UICC), ICC staging is identical to that of hepatocellular carcinoma [8]. However, ICC has different carcinogenic mechanisms and biological behavior from hepatocellular carcinoma. Therefore, it was established as its own category in the revised staging system in the AJCC 7th edition staging manual [9], which is mainly derived from research conducted by Nathan et al. [10]. In the 7th edition staging system, the tumor growth patterns of the PI-type were first mentioned as the definition of primary tumor $4(\mathrm{~T} 4)$. After that, more research about 
the PI-type of ICC was reported. Uno M et al. [11] found that the percentage of intrahepatic metastases in ICC-PI patients was significantly lower than ICC-MF patients and that surgery could provide a more favorable outcome in ICC-PI patients. Imai K et al. [12] reported that patients with the PI-type of ICC without hilar invasion tend to have favorable surgical outcomes than patients with the MF-type. In contrast, Dover LL et al. [13] reported that patients with the MF-type of ICC exhibited significantly better survival than patients with the PI-type of ICC after surgical resection. Another study about the prognostic value of T4 (the tumor growth pattern of PI-type) was also reported. In 233 ICC patients who underwent curative resection, Takahiro Uenishi et al. [14] found that the survival curves failed to stratify the patients according to the 7 th edition AJCC/UICC T classification because the survival prognoses of T2, T3, and T4 tumors were similar. Therefore, in the 8th edition staging system [7], the T4 category, which described the PI-type of ICC, was eliminated due to the controversial prognostic value of the growth pattern $[6,15-17]$. The differences between the AJCC 6th, 7th and 8th edition staging manuals are described in Table 1.

Because the mechanisms of carcinogenesis may differ among these macroscopic types [18], the MF-type of ICC often invades into the adjacent liver parenchyma, whereas the PI-type of ICC invades into the hepatic hilum. The growth and invasive patterns between these subtypes were all different. The present study was conducted to analyze differences in the clinicopathological factors and survival prognoses between the MF-type and PI-type of ICC using a large data set and to evaluate the viability of the AJCC 8th edition staging classification for the MF-type and PI-type. Finally, we proposed some modifications for the AJCC 8th edition staging system to render it more suitable for distinguishing the MF-type and PI-type of ICC.

\section{RESULTS}

\section{Patient characteristics}

In total, 2,679 patients from the SEER database with either pathologically or clinically confirmed ICC were included in this study (Table 2), including 199 cases of the ICC-PI subtype and 2,480 cases of the ICC-MF subtype. The median age at diagnosis was 67.0 years (range: 15-99 years), and the median CSS and OS were 10.1 and 9.4 months (range: 0-59 months for both), respectively. The comparison of clinicopathological characteristics of the ICC-PI and ICC-MF subtypes are summarized in Table 2. There were some differences in the characteristics between the two types, including race, AJCC stage (8th edition, 2017), and surgical status. Thus, we used the intergroup analysis to define the specific differences in the race and AJCC stage subgroups. The results indicated that higher percentage of black patients with ICC presented the
PI-type the than MF-type (14.1\% vs. 7.7\%, respectively; $P<0.001)$. In addition, ICC-MF type patients represented a significantly higher percentage of AJCC stage II cases (21.5\% vs. $14.1 \%, P=0.013)$ and a lower percentage of AJCC stage IV cases $(34.3 \%$ vs. $41.7 \%, P=0.036)$. Moreover, ICC-PI type patients were more inclined to undergo surgery than ICC-MF type patients $(33.7 \%$ vs. 26.3, $P=0.029)$. Other tumor characteristics, including age, sex, marital status, histologic grade, node stage, showed similar distributions between the two subtypes.

\section{Comparison of survival between the ICC-MF type and ICC-PI type}

There were no differences between the ICC-MF type and ICC-PI type regarding the survival prognosis. Figure 1 presents the Kaplan-Meier survival curves of CSS and OS for the two macroscopic types. The CSS (log-rank, $P=0.625)$ and OS (log-rank, $P=0.628)$ between these two ICC subtypes were similar.

Table 3 summarize the prognostic factors according to the CSS and OS results from multivariate Cox proportional hazard regression models for these two macroscopic types. As the AJCC staging system predominantly comprises node stages, we did not include node-stage variables in the multivariate Cox model because of their obvious correlation. Many factors, including older age, poor differentiation, and lack of surgical treatment, were all significantly associated with poor CSS and OS in the multivariate Cox analysis for these two macroscopic types (Table 3). After conducting subgroup analyses for the macroscopic subtypes using multivariate Cox models, we found that statistically significant differences existed among all the AJCC stages for ICC-MF patients according to CSS and OS. From Figure 2, the survival curves were also well differentiated by AJCC stage for ICC-MF patients. However, in ICCPI type patients, the AJCC stage was no longer an independent prognostic factor for CSS and OS. It is also notable that the AJCC staging classification for ICCPI patients overlapped between stage I and II disease according to CSS (log-rank, $P=0.762$ ) and OS (log-rank, $P=0.972$ ) (Figure 3).

\section{Stratification analysis with AJCC stage subtype}

We stratified the AJCC stage subtype to further validate the different outcomes affected by AJCC stage subtype between ICC-MF and ICC-PI cases. As shown in Table 4, the multivariate analysis revealed similar survival for AJCC stage II, III, and IV between ICC-MF and ICC-PI cases. However, in AJCC stage I cases, survival was poor in ICC-PI patients compared to that in ICC-MF patients according to CSS (with ICC-MF as the reference value: HR for ICC-PI, HR: 1.941, $P=0.014$ ) and OS (HR: $1.985, P=0.007)$. When we compared ICC-PI patients 
Table 1: Different AJCC staging definitions for intrahepatic cholangiocarcinoma based on the AJCC 6th edition (2004), AJCC 7th edition (2010) and AJCC 8th edition (2017) staging systems

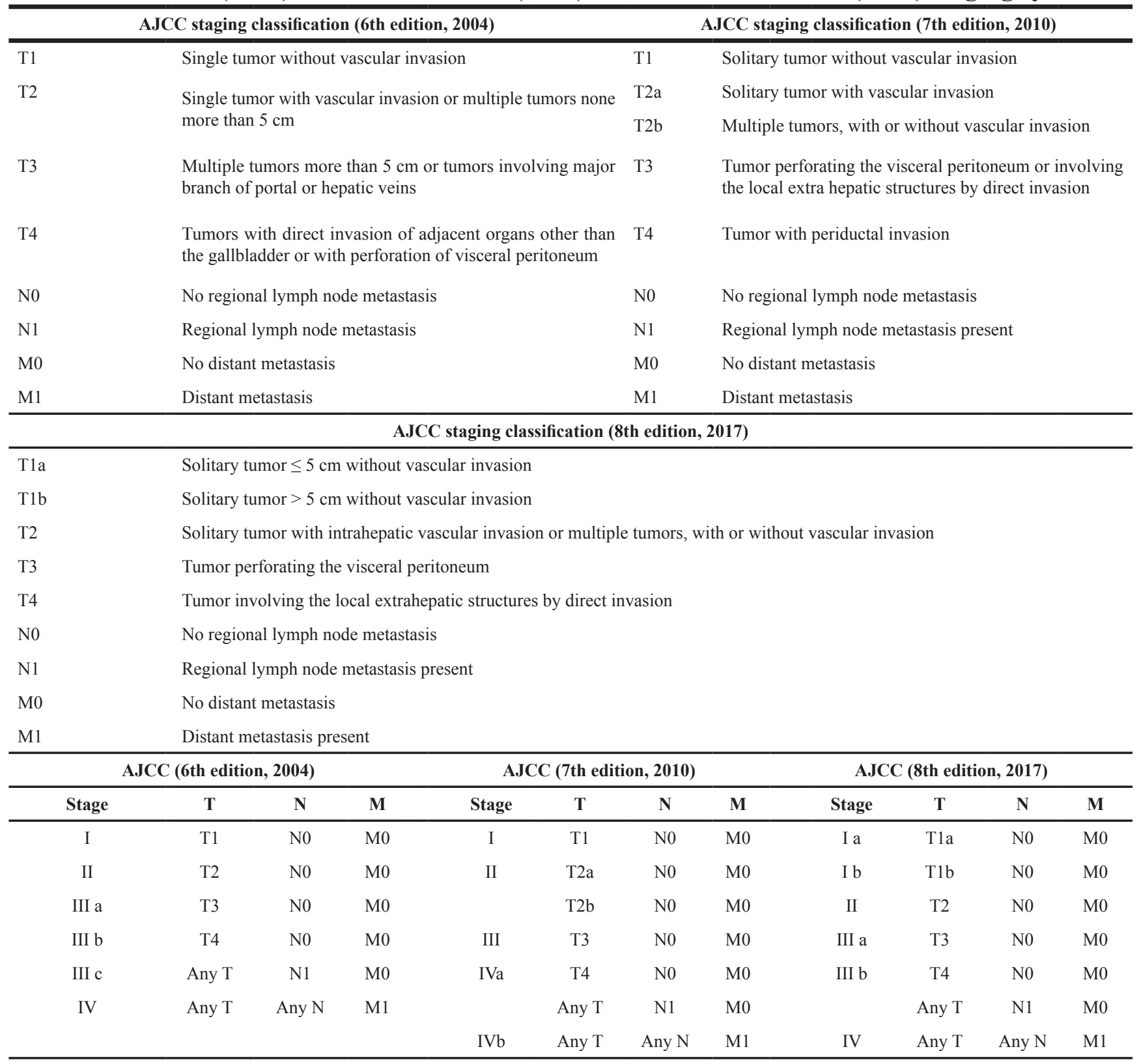

Abbreviations: AJCC, American Joint Committee on Cancer.

in AJCC stage I to ICC-MF patients in AJCC stage II, there was no difference in survival according to CSS (with ICC-MF in stage II as the reference value: HR for ICC-PI in stage I, HR: $1.208, P=0.475)$ and OS (HR: 1.206 , $P=0.456)$. These findings indicated that ICC-PI patients in stage I has a similar survival prognosis as ICC-PI and ICC-MF patients in stage II.

\section{DISCUSSION}

The incidence of ICC is increasing, and the prognosis of ICC patients remains unfavorable. Since the recent release of the 8 th edition of the AJCC staging system, there have been no reports on the advantage and applicability of this staging system. Using populationbased data from a large cohort, we aimed to analyze the characteristics and outcomes of ICC-PI and ICC-MF patients and to evaluate the viability of the AJCC 8th edition staging classification for the MF and PI subtypes of ICC. The purpose of this study was to provide suggestions for staging ICC-MF and ICC-PI patients.

In total, 2,679 ICC patients were included in this study, 199 (7.4\%) of which were had the PI subtype of ICC. This proportion was comparable to other studies $[11,15]$. Table 2 shows that the PI-type of ICC is more prominent among black Americans, which is a new 
Table 2: Clinicopathological characteristics of patients with the MF-type and PI-type of intrahepatic cholangiocarcinoma

\begin{tabular}{|c|c|c|c|c|}
\hline \multirow{2}{*}{ Characteristic } & MF-type $(n=2480)$ & PI-type $(n=199)$ & Total $(n=2679)$ & \multirow{2}{*}{$\mathbf{P}^{\mathrm{a}}$} \\
\hline & No (\%) & No $(\%)$ & No (\%) & \\
\hline Age (years) & & & & 0.878 \\
\hline$\leq 60$ & $753(30.4)$ & $62(31.2)$ & $815(30.4)$ & \\
\hline$>60$ & $1727(69.6)$ & $137(68.8)$ & $1864(69.6)$ & \\
\hline Race & & & & 0.005 \\
\hline White & $1942(78.3)$ & 149 (74.9) & $2091(78.1)$ & \\
\hline Black & $190(7.7)$ & $28(14.1)$ & $218(8.1)$ & \\
\hline Other $^{\mathrm{b}}$ & $348(14.0)$ & $22(11.0)$ & $370(13.8)$ & \\
\hline Sex & & & & 0.878 \\
\hline Male & $1425(57.5)$ & $116(58.3)$ & $1541(57.5)$ & \\
\hline Female & $1055(42.5)$ & $83(41.7)$ & $1138(42.5)$ & \\
\hline Marital status & & & & 0.350 \\
\hline Married & $1442(58.1)$ & $123(61.8)$ & $1565(58.4)$ & \\
\hline Not married ${ }^{c}$ & $1038(41.9)$ & $76(38.2)$ & $1114(41.6)$ & \\
\hline Histologic grade & & & & 0.152 \\
\hline Grade I & $123(5.0)$ & $4(2.0)$ & $127(4.7)$ & \\
\hline Grade II & $517(20.8)$ & $52(26.1)$ & $569(21.2)$ & \\
\hline Grade III & $463(18.7)$ & $38(19.1)$ & $501(18.7)$ & \\
\hline Grade IV & $9(0.4)$ & $1(0.5)$ & $10(0.5)$ & \\
\hline Unknown & $1368(55.1)$ & $104(52.3)$ & $1472(54.9)$ & \\
\hline $\begin{array}{l}\text { AJCC stage } \\
\text { (8th edition, 2017) }\end{array}$ & & & & 0.043 \\
\hline Stage I & $597(24.1)$ & $48(24.1)$ & $645(24.1)$ & \\
\hline Stage II & $533(21.5)$ & $28(14.1)$ & $561(20.9)$ & \\
\hline Stage III & $498(20.1)$ & $40(20.1)$ & $538(20.1)$ & \\
\hline Stage IV & $852(34.3)$ & $83(41.7)$ & $935(34.9)$ & \\
\hline Node stage & & & & 0.979 \\
\hline N0 & $1677(67.6)$ & $133(66.8)$ & $1810(67.6)$ & \\
\hline N1 & $706(28.5)$ & $57(28.7)$ & $763(28.5)$ & \\
\hline NX & $97(3.9)$ & $9(4.5)$ & $106(3.9)$ & \\
\hline Surgery performed & & & & 0.029 \\
\hline Yes & $652(26.3)$ & $67(33.7)$ & $719(26.8)$ & \\
\hline No & $1828(73.7)$ & $132(66.3)$ & $1960(73.2)$ & \\
\hline
\end{tabular}

Abbreviations: AJCC, American Joint Committee on Cancer. MF, mass-forming; PI, periductal infiltrating; NX: Regional lymph nodes could not be assessed.

${ }^{a}$ Bold type indicates statistical significance.

${ }^{b}$ Other includes Asian/Pacific Islander and American Indian/Alaskan native.

'Not married includes single, divorced, separated, unmarried or domestic partner, and widowed.

discovery. In addition, the percentage of the ICC-PI type cases at AJCC stage II was lower than that of the ICC-MF type cases. However, the proportion of ICC-PI type cases in AJCC stage IV was higher than that of ICC-MF type cases. This translates as fewer intrahepatic metastases but more distant metastases in the ICC-PI type. The characteristic of a high rate of distant metastasis for the ICC-PI type has not been reported to date. Moreover, ICC-PI type patients were more inclined to accept surgery than ICC-MF type patients - this is probably due to the 
Table 3: Multivariate Cox analysis of cancer-specific survival and overall survival for the different growth patterns of intrahepatic cholangiocarcinoma

\begin{tabular}{|c|c|c|c|c|c|c|c|c|c|c|c|c|}
\hline \multirow{3}{*}{ Characteristic } & \multicolumn{6}{|c|}{ Cancer-specific survival } & \multicolumn{6}{|c|}{ Overall survival } \\
\hline & \multicolumn{3}{|c|}{ MF-type } & \multicolumn{3}{|c|}{ PI-type } & \multicolumn{6}{|c|}{ PI-type } \\
\hline & HR & $95 \%$ CI & $\mathbf{p}^{\mathrm{a}}$ & HR & $95 \% \mathrm{CI}$ & $\mathbf{p}^{\mathrm{a}}$ & HR & $95 \%$ CI & $\mathbf{p}^{\mathrm{a}}$ & HR & $95 \%$ CI & $\mathbf{p}^{\mathrm{a}}$ \\
\hline \multicolumn{13}{|l|}{ Age (years) } \\
\hline$\leq 60$ & 1 & & & 1 & & & 1 & & & 1 & & \\
\hline$>60$ & 1.505 & $1.260-1.798$ & $<0.001$ & 1.888 & $1.029-3.463$ & 0.040 & 1.543 & $1.299-1.834$ & $<0.001$ & 2.058 & $1.127-3.757$ & 0.019 \\
\hline \multicolumn{13}{|l|}{ Race } \\
\hline White & 1 & & & 1 & & & 1 & & & 1 & & \\
\hline Black & 1.243 & $0.913-1.691$ & 0.168 & 1.078 & $0.503-2.311$ & 0.846 & 1.210 & $0.895-1.636$ & 0.215 & 1.008 & $0.474-2.142$ & 0.984 \\
\hline Other & 0.942 & $0.742-1.197$ & 0.626 & 1.235 & $0.546-2.790$ & 0.612 & 0.989 & $0.789-1.240$ & 0.925 & 1.364 & $0.627-2.969$ & 0.434 \\
\hline \multicolumn{13}{|l|}{ Sex } \\
\hline Male & 1 & & & 1 & & & 1 & & & 1 & & \\
\hline Female & 0.887 & $0.748-1.053$ & 0.171 & 0.804 & $0.451-1.432$ & 0.458 & 0.880 & $0.746-1.038$ & 0.130 & 0.710 & $0.404-1.247$ & 0.233 \\
\hline \multicolumn{13}{|l|}{ Marital status } \\
\hline Married & 1 & & & 1 & & & 1 & & & 1 & & \\
\hline Not married & 1.093 & $0.922-1.296$ & 0.303 & 0.838 & $0.467-1.504$ & 0.554 & 1.112 & $0.944-1.310$ & 0.202 & 0.842 & $0.477-1.485$ & 0.552 \\
\hline \multicolumn{13}{|l|}{ Histologic grade } \\
\hline Grade I + II & 1 & & & 1 & & & 1 & & & 1 & & \\
\hline Grade III + IV & 1.574 & $1.333-1.859$ & $<0.001$ & 2.583 & $1.483-4.499$ & 0.001 & 1.560 & $1.329-1.832$ & $<0.001$ & 2.398 & $1.400-4.107$ & 0.001 \\
\hline \multicolumn{13}{|l|}{$\begin{array}{l}\text { AJCC stage } \\
\text { (8th edition, 2017) }\end{array}$} \\
\hline Stage I & 1 & & & 1 & & & 1 & & & 1 & & \\
\hline Stage II & 1.766 & $1.341-2.326$ & $<0.001$ & 0.969 & $0.371-2.534$ & 0.949 & 1.760 & $1.356-2.285$ & $<0.001$ & 0.832 & $0.323-2.141$ & 0.703 \\
\hline Stage III & 2.176 & $1.657-2.858$ & $<0.001$ & 1.837 & $0.847-3.987$ & 0.124 & 2.073 & $1.597-2.691$ & $<0.001$ & 1.543 & $0.729-3.267$ & 0.257 \\
\hline Stage IV & 2.397 & $1.846-3.113$ & $<0.001$ & 1.971 & $0.864-4.496$ & 0.107 & 2.315 & $1.804-2.971$ & $<0.001$ & 2.018 & $0.909-4.478$ & 0.084 \\
\hline \multicolumn{13}{|l|}{ Surgery performed } \\
\hline Performed & 1 & & & 1 & & & 1 & & & 1 & & \\
\hline Not performed & 4.115 & $3.321-5.098$ & $<0.001$ & 2.897 & $1.424-5.891$ & 0.003 & 3.938 & $3.210-4.830$ & $<0.001$ & 2.784 & $1.392-5.569$ & 0.004 \\
\hline
\end{tabular}

Abbreviations: AJCC, American Joint Committee on Cancer; MF, mass-forming; PI, periductal infiltrating.

${ }^{a}$ Bold type indicates statistical significance.
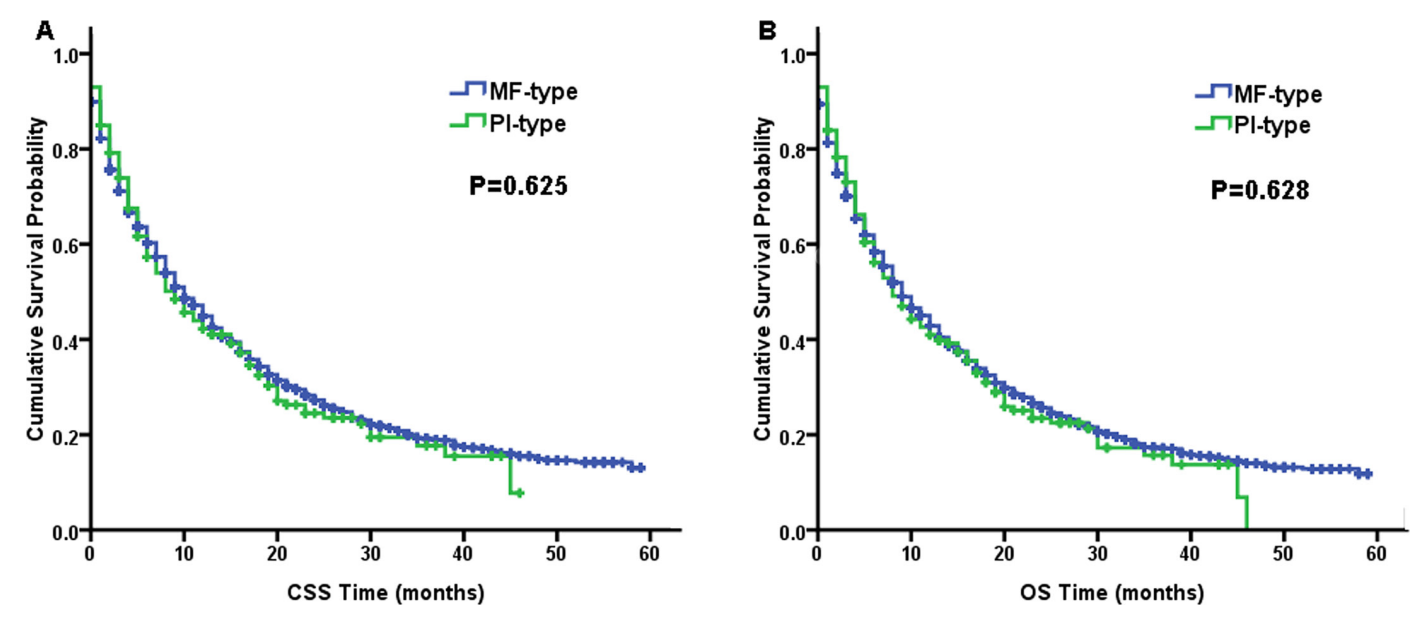

Figure 1: Kaplan-Meier curves of cancer-specific survival (CSS, A) and overall survival (OS, B) for the mass-forming type (MF-type) vs. periductal infiltrating type (PI-type) of intrahepatic cholangiocarcinoma. 
Table 4: Comparison of cancer-specific survival and overall survival between the MF-type and PI-type of intrahepatic cholangiocarcinoma after subgroup analyses using a multivariate Cox proportional hazard model

\begin{tabular}{|c|c|c|c|c|c|c|}
\hline \multirow{2}{*}{$\begin{array}{l}\text { AJCC stage } \\
\text { (8th edition, 2017) }\end{array}$} & \multicolumn{3}{|c|}{ Cancer-specific survival } & \multicolumn{3}{|c|}{ Overall survival } \\
\hline & HR & $95 \% \mathrm{CI}$ & $\mathbf{p}^{\mathrm{a}}$ & HR & $95 \% \mathrm{CI}$ & $\mathbf{p}^{\mathrm{a}}$ \\
\hline \multicolumn{7}{|l|}{ stage I } \\
\hline MF-type $(n=597)$ & 1 & & & 1 & & \\
\hline PI-type $(n=48)$ & 1.941 & $1.141-3.302$ & 0.014 & 1.985 & $1.202-3.280$ & 0.007 \\
\hline \multicolumn{7}{|l|}{ stage II } \\
\hline MF-type $(n=533)$ & 1 & & & 1 & & \\
\hline PI-type $(n=28)$ & 1.188 & $0.515-2.739$ & 0.687 & 1.038 & $0.452-2.383$ & 0.931 \\
\hline \multicolumn{7}{|l|}{ stage III } \\
\hline MF-type $(n=498)$ & 1 & & & 1 & & \\
\hline PI-type $(n=40)$ & 1.296 & $0.728-2.307$ & 0.378 & 1.214 & $0.685-2.153$ & 0.506 \\
\hline \multicolumn{7}{|l|}{ stage IV } \\
\hline MF-type $(n=852)$ & 1 & & & 1 & & \\
\hline PI-type $(n=83)$ & 1.085 & $0.714-1.650$ & 0.702 & 1.104 & $0.737-1.652$ & 0.632 \\
\hline \multicolumn{7}{|l|}{ MF of stage II vs. PI of stage I } \\
\hline stage II $\quad$ MF-type $(n=533)$ & 1 & & & 1 & & \\
\hline stage I $\quad$ PI-type $(n=48)$ & 1.208 & $0.719-2.031$ & 0.475 & 1.206 & $0.737-1.972$ & 0.456 \\
\hline
\end{tabular}

Abbreviations: AJCC, American Joint Committee on Cancer; MF, mass-forming; PI, periductal infiltrating.

aThe $P$ value was adjusted using a multivariate Cox proportional hazard regression model that accounted for age, race, sex, marital status, histologic grade, and surgery status. Bold type indicates statistical significance.

lower incidence of intrahepatic metastases in the ICC-PI type [11].

Based on the results of this study, we found that the age, histologic grade, AJCC stage (8th edition), and surgical status were related prognostic factors (Table 3). This finding was previously reported in many other studies [19-21]. However, for the ICC-MF type and ICC-

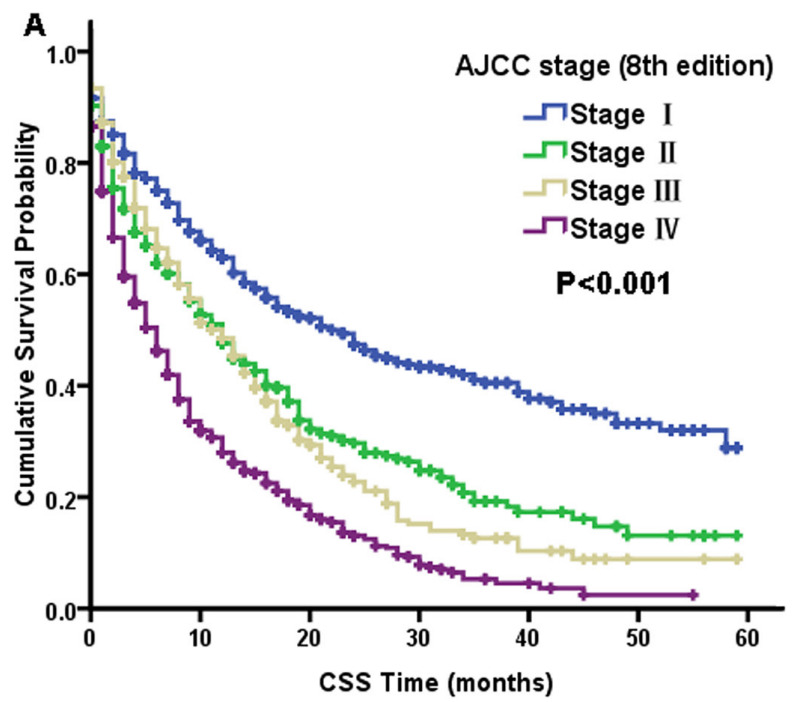

PI type, there were no significant associations with CSS and OS in the ICC patients. Figure 1 presents the survival curves of the two macroscopic types for CSS (log-rank, $P=0.625)$ and OS (log-rank, $P=0.628)$, both of which were similar. But in many other studies, the survival comparison results of the two macroscopic types were various. Some reported that the two macroscopic types

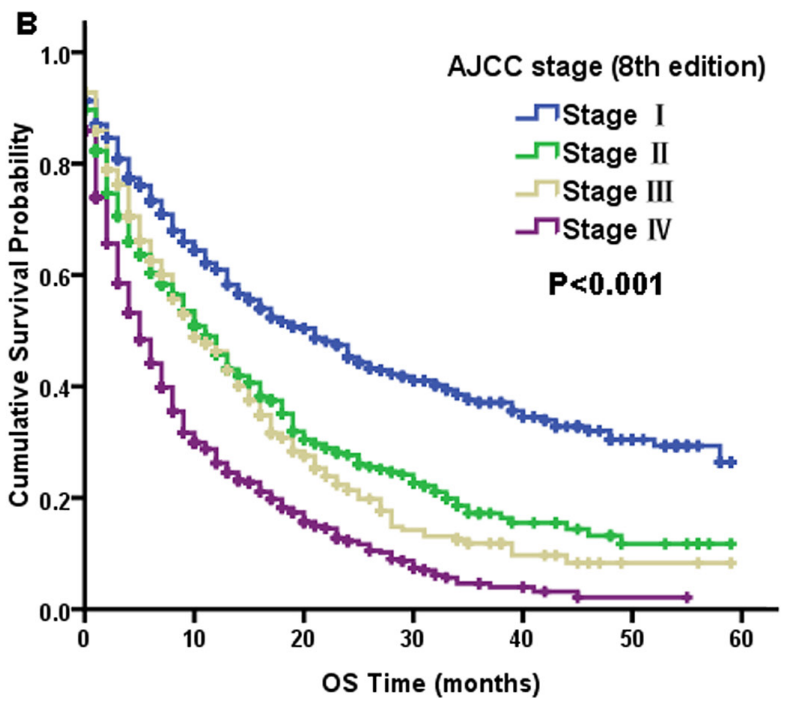

Figure 2: Kaplan-Meier curves of cancer-specific survival (CSS, A) and overall survival (OS, B) based on the AJCC stage of the massforming type (MF-type) of intrahepatic cholangiocarcinoma. 
of ICC have similar survival rates just like our study [6]. In addition, some reported that patients with the PItype of ICC had significantly better survival than those with the MF-type of ICC $[11,12]$. Moreover, there was also a report about the opposite result [13]. The different outcomes of these studies may be due to the characteristics of the ICC-PI type: fewer intrahepatic metastases but more distant metastases. ICC-PI type patients were more inclined to accept surgery than ICC-MF type patients, which was probably due to the lower incidence of intrahepatic metastases in the ICC-PI type [11]. Although we performed radical resection for localized ICC, the tumor may have distantly metastasized, which was not identified prior to surgery. This confounding factor leads to the unpredictable survival rates after surgery and nonsensical result above. For our study, the survival between the PI-type and the MF-type of ICC was similar in the entire ICC patient cohort.

After subgroup analysis for the macroscopic types using multivariate Cox models, we found that the AJCC 8th edition staging system was suitable for staging ICC-MF patients according to CSS and OS (Figure 2, Table 3). However, for ICC-PI patients, the AJCC staging system was no longer an independent prognostic factor for CSS and OS (Table 3). The main reason for this was the similar HR for CSS and OS between the stage I and stage II of ICC-PI patients. Figure 3 shows that the AJCC staging classification overlapped between stage I and II disease according to CSS and OS for ICC-PI patients. Therefore, we stratified the AJCC stage subtypes to further validate the different outcomes affected by AJCC stage subtype between ICC-MF and ICC-PI cases. As shown in Table 4, the multivariate analysis of AJCC stage I cases revealed that ICC-PI patients had poorer survival than ICC-

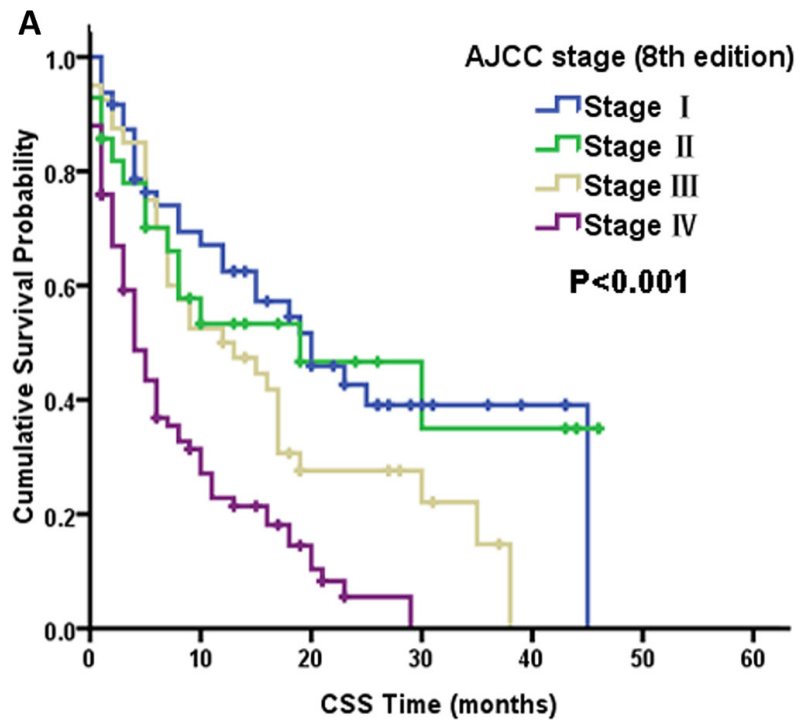

MF patients according to CSS and OS. In addition, we found that the ICC-PI patient group in AJCC stage I disease had a similar HR as the ICC-MF patient group with AJCC stage II disease according to CSS and OS. These findings indicated that ICC-PI patients in stage I have a similar survival prognosis as ICC-PI and ICCMF patients in stage II. The definition of stage $I$ is a solitary tumor without vascular invasion. The main differences between stage I and stage II are vascular invasion and intrahepatic metastasis. Therefore, the staging system is suitable for ICC-MF patients due to its metastasizing characteristics of this subtype via the portal vein system [22]. However, the PI-type of ICC most commonly spreads via the lymphatic system [22]. Therefore, the definitions of AJCC stage I and stage II are not suitable for staging ICC-PI patients, which leads to similar survival prognoses between ICC-PI patients in stage I and ICC-PI and ICC-MF patients in stage II. Because ICC-PI patients in stage I have a similar survival prognosis as ICC disease exhibiting vascular invasion and intrahepatic metastasis (i.e., AJCC stage II) and the uncertainty of its own metastasis behavior, we suggest that the ICC-PI type be defined as a special type of intrahepatic metastasis and be categorized as stage T2 regardless of the presence or absence of intrahepatic metastasis. If a large-scale multicenter is conducted, it should be feasible to establish separate stages for ICCPI due to its different growth and invasive behaviors.

This study is the first to evaluate the viability of the AJCC 8th edition staging classification for the MF-type and PI-type of ICC. In addition, we also propose a slight modification for staging the ICC-PI type in the AJCC 8th edition staging system. However, this study was limited by its retrospective nature, and the results need to be confirmed using additional large-scale studies.

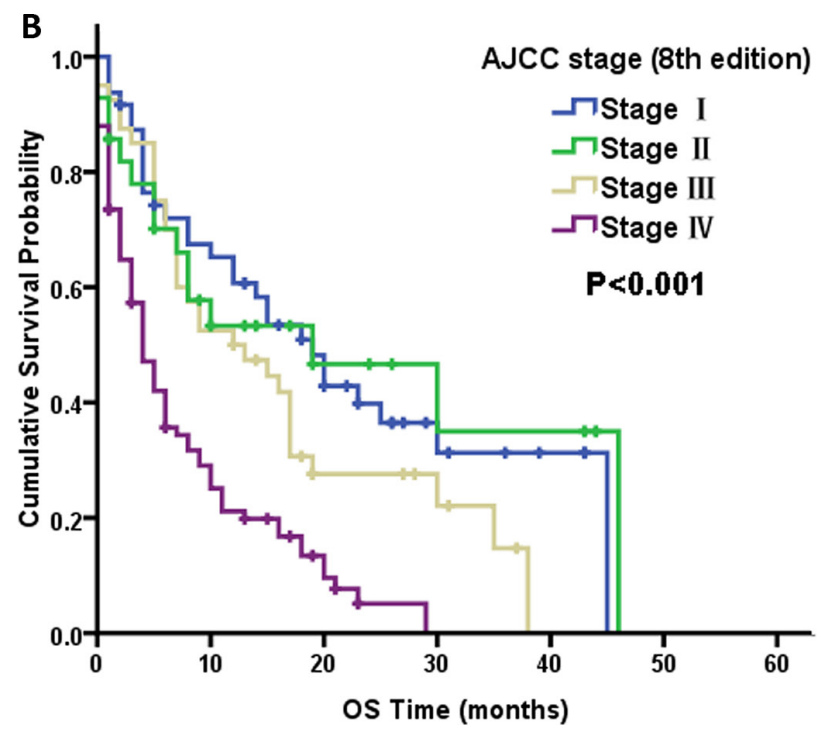

Figure 3: Kaplan-Meier curves of cancer-specific survival (CSS, A) and overall survival (OS, B) based on the AJCC stage of the periductal infiltrating type (PI-type) of intrahepatic cholangiocarcinoma. 
Table 5: The corresponding stage definitions for intrahepatic cholangiocarcinoma from the AJCC 6th edition (2004) and AJCC 7th edition (2010) and AJCC 8th edition (2017)

\begin{tabular}{ccc}
\hline & AJCC staging classification & \\
\hline 8th edition (2017) & 7th edition (2010) & 6th edition (2004) \\
\hline I & I & I \\
II & II & II + III a \\
III & III + IV a-T4N0M0 & III b + III c \\
IV & IV b & IV \\
\hline
\end{tabular}

Abbreviations: AJCC, American Joint Committee on Cancer.

\section{MATERIALS AND METHODS}

\section{Patients}

The Surveillance, Epidemiology, and End Results (SEER) [23] database (1973 to 2014) was used to identify ICC patients. Patients evaluated between 2010 and 2014 were chosen because both the 6th and 7th editions of the AJCC staging system were used to characterize the patients examined during this period. Since the release of the 8th edition of the AJCC staging system in 2017, it has not yet implemented in a large cohort of clinical patients. We used the data from the AJCC 6th and 7th editions staging classifications to transform to the AJCC 8th edition staging classification to correlate the stages. The corresponding conversion method is described in Table 5. Patients were identified based on the International Classification of Diseases for Oncology (3rd editions) [24]. The following coding was used: the primary site code for the liver (22.0); the histology code for cholangiocarcinoma (8160); the primary site code for the intrahepatic bile duct (22.1); the histology codes for malignant neoplasm (8000), malignant tumor cells (8001), carcinoma (8010), undifferentiated carcinoma (8020), adenocarcinoma (8140), and cholangiocarcinoma (8160); and a behavior code (3-malignant tumor). Finally, 2,679 cases were included in our study. The SEER 8.3.4 registry research database was utilized to generate a listing of ICC cases, and the following variables were extracted: site recode (intrahepatic bile duct), behavior recode for analysis (malignant), age, race, sex, marital status at diagnosis, growth patterns (i.e., MF-type, PI-type), histological grade, AJCC stage (6th edition, 2004), AJCC stage (7th edition, 2010), node stage, surgical status (yes, no), SEER cause-specific death classification, vital status recode, and survival (months). Age at diagnosis was stratified as $\leq 60$ years and $>60$ years. Race was recoded as white, black, or other (includes Asian/Pacific Islander and American Indian/Alaskan native). Marital status was categorized as married or not married (i.e., single, divorced, separated, unmarried or domestic partner, widowed). Tumor growth patterns were classified as MF-type and PItype. Histological grades were classified as grade I (well differentiated), grade II (moderately differentiated), grade
III (poorly differentiated), grade IV (undifferentiated), and unknown. The cancer-specific survival (CSS) was defined as the time from the date of diagnosis to the date of either death due to ICC, and overall survival (OS) was calculated from the date of diagnosis to the date of death due to any cause. Patients who were alive were censored on the date of last contact for both outcomes. The characteristics of the 2,679 patients with ICC between the different growth patterns are described in Table 2.

\section{Statistical analysis}

All computations were performed using SPSS version 13.0 for Windows (SPSS Inc, IL, USA). The chi-square test and one-way analysis of variance were employed to compare the demographic and clinical characteristics of the ICC-MF and ICC-PI groups. CSS and OS were analyzed using Kaplan-Meier curves, and log-rank tests were used to evaluate the correlation between the AJCC 8th edition staging system and growth patterns. Multivariate analyses for each staging system and different growth patterns were completed using Cox proportional hazards regression models controlling for growth patterns, age, race, sex, marital status at diagnosis, histological grade, and surgical status. The hazard ratio (HR) and 95\% confidence interval (CI) were calculated. All $P$ values were two-sided, and values less than 0.05 were considered statistically significant.

\section{Ethics statement}

For access to the SEER database, informed consent was not required, but a Data-Use Agreement for the SEER 1973-2014 Research Data File was completed.

\section{Abbreviations}

ICC: intrahepatic cholangiocarcinoma; MF, massforming; PI: periductal infiltrating; AJCC: American Joint Committee on Cancer; UICC: Union for International Cancer Control; TNM: tumor-node-metastasis; CSS: cancer-specific survival; OS: overall survival; SEER: Surveillance, Epidemiology, and End Results; HR, hazard ratio; CI: confidence interval. 


\section{Author contributions}

Conception and design: Ze-Wu Meng; Financial support: Ze-Wu Meng, Yan-Ling Chen; Collection and assembly of data: All authors; Data analysis and interpretation: Ze-Wu Meng, Wei Pan, Hai-Jie Hong, Jiang-Zhi Chen; Manuscript writing: Ze-Wu Meng; Final approval of manuscript: All authors; Accountable for all aspects of the work: All authors.

\section{CONFLICTS OF INTEREST}

The authors have declared that no competing interests exist.

\section{FUNDING}

This study was supported by the National Clinical Key Specialty Construction Project (General Surgery) of China, the MiaoPu Youth Fund in Fujian Medical University (no. 2015MP002), and the Youth Fund of Fujian Provincial Health and Family Planning Commission (no. 2016-1-48).

\section{REFERENCES}

1. West J, Wood H, Logan RF, Quinn M, Aithal GP. Trends in the incidence of primary liver and biliary tract cancers in England and Wales 1971-2001. Br J Cancer. 2006; 94:1751-1758.

2. Welzel TM, McGlynn KA, Hsing AW, O'Brien TR, Pfeiffer RM. Impact of classification of hilar cholangiocarcinomas (Klatskin tumors) on the incidence of intra- and extrahepatic cholangiocarcinoma in the United States. J Natl Cancer Inst. 2006; 98:873-875.

3. Bergquist A, von Seth E. Epidemiology of cholangiocarcinoma. Best Pract Res Clin Gastroenterol. 2015; 29:221-232.

4. Zhang $\mathrm{H}$, Yang $\mathrm{T}$, Wu $\mathrm{M}$, Shen $\mathrm{F}$. Intrahepatic cholangiocarcinoma: Epidemiology, risk factors, diagnosis and surgical management. Cancer Lett. 2016; 379:198-205.

5. Meng ZW, Han SH, Zhu JH, Zhou LY, Chen YL. Risk Factors for Cholangiocarcinoma After Initial Hepatectomy for Intrahepatic Stones. World J Surg. 2017; 41:835-843.

6. Hirohashi K, Uenishi T, Kubo S, Yamamoto T, Tanaka H, Shuto T, Kinoshita H. Macroscopic types of intrahepatic cholangiocarcinoma: clinicopathologic features and surgical outcomes. Hepatogastroenterology. 2002; 49:326-329.

7. Amin MB, Greene FL, Edge SB, Compton CC, Gershenwald JE, Brookland RK, Meyer L, Gress DM, Byrd DR, Winchester DP. The Eighth Edition AJCC Cancer Staging Manual: Continuing to build a bridge from a population-based to a more "personalized" approach to cancer staging. CA Cancer J Clin. 2017; 67:93-99.
8. Greene FL. American Joint Committee on Cancer, American Cancer Society. AJCC cancer staging manual. 6th ed. New York: Springer-Verlag. 2002.

9. Edge SB. American Joint Committee on Cancer, American Cancer Society. AJCC cancer staging manual. 7th ed. New York: Springer. 2009.

10. Nathan H, Aloia TA, Vauthey JN, Abdalla EK, Zhu AX, Schulick RD, Choti MA, Pawlik TM. A proposed staging system for intrahepatic cholangiocarcinoma. Ann Surg Oncol. 2009; 16:14-22.

11. Uno M, Shimada K, Yamamoto Y, Nara S, Esaki M, Sakamoto Y, Kosuge T, Ojima H. Periductal infiltrating type of intrahepatic cholangiocarcinoma: a rare macroscopic type without any apparent mass. Surg Today. 2012; 42:1189-1194.

12. Imai K, Yamamoto M, Ariizumi S. Surgery for periductal infiltrating type intrahepatic cholangiocarcinoma without hilar invasion provides a better outcome than for massforming type intrahepatic cholangiocarcinoma without hilar invasion. Hepatogastroenterology. 2010; 57:1333-1336.

13. Dover LL, Jacob R, Wang TN, Richardson JH, Redden DT, Li P, DuBay DA. Improved Postoperative Survival for IntraductalGrowth Subtype of Intrahepatic Cholangiocarcinoma. Am Surg. 2016; 82:1133-1139.

14. Uenishi T, Ariizumi S, Aoki T, Ebata T, Ohtsuka M, Tanaka E, Yoshida H, Imura S, Ueno M, Kokudo N, Nagino M, Hirano S, Kubo S, et al. Proposal of a new staging system for mass-forming intrahepatic cholangiocarcinoma: a multicenter analysis by the Study Group for Hepatic Surgery of the Japanese Society of Hepato-BiliaryPancreatic Surgery. J Hepatobiliary Pancreat Sci. 2014; 21:499-508.

15. Yamasaki S. Intrahepatic cholangiocarcinoma: macroscopic type and stage classification. J Hepatobiliary Pancreat Surg. 2003; 10:288-291.

16. Sanada Y, Kawashita Y, Okada S, Azuma T, Matsuo S. Review to better understand the macroscopic subtypes and histogenesis of intrahepatic cholangiocarcinoma. World J Gastrointest Pathophysiol. 2014; 5:188-199.

17. Ariizumi S, Yamamoto M. Surgical treatment of intrahepatic cholangiocarcinoma based on the macroscopic subtype. [Article in Japanese]. Nihon Shokakibyo Gakkai Zasshi. 2012; 109:1885-1894.

18. Ohashi K, Nakajima Y, Kanehiro H, Tsutsumi M, Taki J, Aomatsu Y, Yoshimura A, Ko S, Kin T, Yagura K. Kiras mutations and $\mathrm{p} 53$ protein expressions in intrahepatic cholangiocarcinomas: relation to gross tumor morphology. Gastroenterology. 1995; 109:1612-1617.

19. Mavros MN, Economopoulos KP, Alexiou VG, Pawlik TM. Treatment and Prognosis for Patients With Intrahepatic Cholangiocarcinoma: Systematic Review and Metaanalysis. JAMA Surg. 2014; 149:565-574.

20. Luo X, Yuan L, Wang Y, Ge R, Sun Y, Wei G. Survival outcomes and prognostic factors of surgical therapy for all potentially resectable intrahepatic cholangiocarcinoma: a 
large single-center cohort study. J Gastrointest Surg. 2014; 18:562-572.

21. Hammad AY, Berger NG, Eastwood D, Tsai S, Turaga KK, Christian KK, Johnston FM, Pawlik TM, Gamblin TC. Is Radiotherapy Warranted Following Intrahepatic Cholangiocarcinoma Resection? The Impact of Surgical Margins and Lymph Node Status on Survival. Ann Surg Oncol. 2016; 23:912-920.

22. Sasaki A, Aramaki M, Kawano K, Morii Y, Nakashima $\mathrm{K}$, Yoshida $\mathrm{T}$, Kitano $\mathrm{S}$. Intrahepatic peripheral cholangiocarcinoma: mode of spread and choice of surgical treatment. Br J Surg. 1998; 85:1206-1209.
23. Institute. NC. SEER: Surveillance, Epidemiology, and End Results Program. http://seer.cancer.gov.Accessed. November, 2014.

24. Fritz PC, Jack A, Shanmugaratnam K, Sobin L, Parkin WS. International Classification of Diseases for Oncology. 3rd ed. Geneva, Switzerland: World Health Organization; 2000. 